\title{
Custo e eficiência da técnica de múltipla ovulação e transferência de embriões em ovinos ao longo de quatro anos: Influência da capacitação do médico-veterinário
}

\author{
Cost and efficiency of multiple ovulation and embryo transfer technique in sheep during four years: \\ Influence of the veterinary capacitation \\ Costo y eficiencia de la técnica de ovulación múltiple y transferencia embrionaria en ovinos durante \\ cuatro años: Influencia de la capacitación veterinaria
}

Recebido: 16/07/2021 | Revisado: 20/07/2021 | Aceito: 21/07/2021 | Publicado: 30/07/2021

\author{
Marcela Sene Rocha \\ ORCID: https://orcid.org/0000-0002-3505-8021 \\ Universidade de São Paulo, Brasil \\ E-mail: marcela_sene@hotmail.com \\ Paulo Victor dos Santos Pereira \\ ORCID: https://orcid.org/0000-0003-4784-9450 \\ Universidade Federal Fluminense, Brasil \\ E-mail: victor_paulo@id.uff.br \\ Paulo Sergio Cerqueira Rangel \\ ORCID: https://orcid.org/0000-0003-3956-9408 \\ Universidade do Grande Rio, Brasil \\ E-mail: paulorangel1102@gmail.com \\ Luiza Carneiro Mareti Valente \\ ORCID: https://orcid.org/0000-0001-8131-632X \\ Universidade Federal Fluminense, Brasil \\ E-mail: 1mareti@id.uff.br \\ Cláudio Alvarenga de Oliveira \\ ORCID: https://orcid.org/0000-0002-4143-7212 \\ Universidade de São Paulo, Brasil \\ E-mail: cadolive@usp.br \\ Joanna Maria Gonçalves Souza-Fabjan \\ ORCID: https://orcid.org/0000-0002-4872-1718 \\ Universidade Federal Fluminense, Brasil \\ E-mail: joannavet@gmail.com
}

\section{Resumo}

A múltipla ovulação e transferência de embriões (MOTE) é a biotecnologia mais utilizada nos rebanhos comerciais ovinos para produção de embriões, porém, vários fatores podem interferir no sucesso e viabilidade financeira da técnica. O objetivo deste estudo foi avaliar o impacto do treinamento do médico-veterinário nos custos e viabilidade financeira desta biotécnica. O estudo foi realizado ao longo de quatro anos (2017-2020) em uma cabanha no estado de SP, Brasil. Ovelhas pluríparas da raça Dorper $(n=35)$ foram submetidas ao programa de MOTE utilizando protocolo convencional (256 mg FSH) por até cinco vezes $(n=65)$. A inseminação artificial aconteceu no dia 16 por laparoscopia e a coleta cirúrgica dos embriões com transferência para as receptoras $(n=140)$ cinco dias após. Foram levantados os custos diretos para realização da MOTE, índices reprodutivos e resultados financeiros da cabanha. A quantidade média de estruturas totais e embriões transferidos por coleta foi superior $(\mathrm{P}<0,05)$ nos últimos anos em relação ao primeiro. Não houve diferença $(\mathrm{P}>0,05)$ nas taxas de gestação, aderência permanente e transitória das doadoras. A margem bruta por gestação foi negativa apenas no primeiro ano e aumentou substancialmente nos anos seguintes, estabilizando a variação anual entre custos e valor de venda dos animais. Em conclusão, o treinamento técnico do médico-veterinário ao longo dos quatro anos foi capaz de diminuir os custos e aumentar a eficiência da MOTE, viabilizando financeiramente o uso da técnica em rebanho ovino da raça Dorper, acelerando o melhoramento genético na cabanha.

Palavras-chave: Biotecnologia; Embrião; Melhoramento genético; MOTE.

\begin{abstract}
The multiple ovulation and embryo transfer (MOET) is the most used biotechnology in commercial sheep herds to produce embryos, however, several factors can interfere with the success and financial viability of the technique. The objective of the work was to evaluate the impact of technical training on the costs and financial feasibility of biotechnology. The study was carried out over four years (2017-2020) in a commercial system, in the state of SP, Brazil. Dorper breed ewes $(n=35)$ were submitted to the MOET program using a conventional protocol (256 mg FSH) for up to five times $(n=65)$. Artificial insemination took place on day 16 by laparoscopy and embryos were surgically collected
\end{abstract}


with transfer to recipients $(n=140)$ five days later. Direct costs for carrying out the MOET, reproductive rates and financial results of the cabanha were raised. The average amount of total structures and embryos transferred per collection showed a significant difference between years $(\mathrm{P}<0.05)$, with no difference $(\mathrm{P}>0.05)$ in the rates of pregnancy, permanent and transient adherence of donors. The gross margin per pregnancy was negative only in the first year and increased substantially in the following years, stabilizing the annual variation between costs and value of animals. In conclusion, the technical training of the veterinarian over four years was able to reduce costs and increase the efficiency of MOET, making the use of the technique financially viable in a Dorper sheep herd, accelerating the genetic improvement in the herd.

Keywords: Biotechnology; Embryo; Genetic improvement; MOET.

\section{Resumen}

La ovulación múltiple y transferencia de embriones (MOET) es la biotecnología más utilizada en los rebaños comerciales de ovejas para la producción de embriones, sin embargo, varios factores pueden interferir con el éxito y la viabilidad financiera de la técnica. El objetivo del trabajo fue evaluar el impacto de la capacitación técnica sobre los costos y la viabilidad financiera de la biotecnología. El estudio se llevó a cabo durante cuatro años (2017-2020) en una cabaña en el estado de SP, Brasil. Se enviaron ovejas de raza Dorper $(\mathrm{n}=35)$ al programa MOET utilizando un protocolo convencional (256 mg de FSH) hasta cinco veces $(\mathrm{n}=65)$. La inseminación artificial se realizó el día 16 por laparoscopia y los embriones fueron extraídos quirúrgicamente con transferencia a los receptores $(\mathrm{n}=140)$ cinco días después. Se elevaron los costos directos para realizar el MOET, las tasas de reproducción y los resultados financieros de la cabaña. La cantidad promedio de estructuras totales y embriones transferidos por colección mostró una diferencia significativa entre años $(\mathrm{P}<0.05)$, sin diferencia $(\mathrm{P}>0.05)$ en las tasas de embarazo, adherencia permanente y transitoria de las donantes. El margen bruto por gestación fue negativo solo en el primer año y aumentó sustancialmente en los años siguientes, estabilizando la variación anual entre costos y valor de los animales. En conclusión, la formación técnica del veterinario a lo largo de cuatro años consiguió reducir costes y aumentar la eficiencia del MOET, haciendo viable económicamente el uso de la técnica en un rebaño de ovejas Dorper, acelerando la mejora genética en el rebaño.

Palabras clave: Biotecnología; Embrión; Mejoramiento genético; MOET.

\section{Introdução}

A ovinocultura é uma importante atividade do agronegócio brasileiro, se estendendo por todo o território nacional (Alves, Osório, Fernandes, Ricardo \& Cunha, 2014). A necessidade crescente do aumento da produtividade animal vem sendo possível por meio da eficiência reprodutiva e melhoramento genético dos animais a partir das biotecnologias da reprodução (Zhu et al., 2018). A múltipla ovulação e transferência de embriões (MOTE) gera grande impacto nos programas de melhoramento genético e disseminação da genética de animais superiores (Brasil, Moreira \& Ramos, 2014). Ressalta-se que o Brasil possui grande destaque na produção mundial de embriões comerciais, sendo o país de maior produção de embriões oriundos da MOTE (IETS, 2020).

Embora a técnica transcervical venha sendo estudada e utilizada mais recentemente (Fonseca et al., 2019), a coleta cirúrgica de embriões ainda é a mais praticada ao redor do mundo. Dentre os entraves para a maior difusão da MOTE estão o custo da técnica, a resposta ao protocolo de superovulação e a manutenção de receptoras sem gestação na propriedade (Heikkila \& Peippo, 2012; Baltrame, Quirino, Barioni \& Lima, 2010; Galli et al., 2003). Os resultados positivos no uso de uma biotecnologia reprodutiva estão diretamente associados à competência e habilidade do técnico que irá realizar tal função (Gerger et al., 2017). Sendo assim, a experiência profissional é um fator essencial para o desenvolvimento de um programa de MOTE bem-sucedido (Phillips \& Janke, 2016) com consequente expansão da técnica e maior retorno financeiro para o produtor (Baruselli et al., 2019).

Apesar das inúmeras vantagens da MOTE, a técnica apresenta um custo relativamente alto que deve ser compensado com elevada efetividade. Avaliações referentes aos custos do uso desta biotécnica e índices obtidos pelo médico-veterinário ao longo de sua capacitação em rebanhos comerciais são escassas, ou mesmo inexistentes, na literatura. O objetivo deste estudo foi avaliar o impacto do treinamento do técnico nos custos e viabilidade financeira da utilização da MOTE em um rebanho comercial ovino.

\section{Metodologia}

\section{1 Ética e local do estudo}

Este estudo foi aprovado no Comitê de Ética no Uso de Animais (CEUA) da Faculdade de Medicina Veterinária e 
Zootecnia da Universidade de São Paulo sob o número 5362181220. O estudo foi realizado em uma cabanha comercial, na cidade

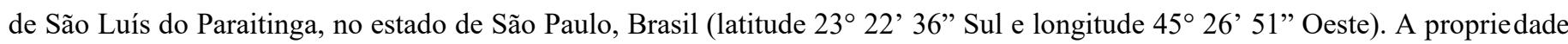
possui animais registrados da raça Dorper com a finalidade de melhoramento genético.

\subsection{Desenho experimental}

Antes da realização do primeiro procedimento de MOTE, o médico-veterinário responsável pela cabanha inicialmente se capacitou por meio de um curso teórico-prático. Foram realizados protocolos de superovulação, inseminação artificial e coleta por via cirúrgica em ovelhas doadoras da raça Dorper e transferência dos embriões para receptoras cruzadas da raça Santa Inês. Todos os procedimentos foram realizados exclusivamente pelo mesmo profissional. Posteriormente, foram levantados todos os custos diretos relacionados ao procedimento. Todas as informações referentes ao uso da biotecnologia e informações financeiras foram registradas ao longo de quatro consecutivos (2017 - 2020), denominados como Ano 1 a 4.

\subsection{Doadoras de embriões}

As 35 ovelhas utilizadas como doadoras possuíam alto valor genético, idade entre dois e nove anos, escore corporal entre 2 e 4 (escala de 1 a 5) e pelo menos um parto antes de serem incluídas como doadoras (primíparas ou pluríparas). Cada animal passou por pelo menos um procedimento de coleta de embriões e no máximo cinco. No total, as fêmeas receberam protocolo de superovulação por 84 vezes, porém apenas as ovelhas $(n=65)$ que responderam ao protocolo foram submetidas à coleta.

\subsection{Protocolo de superovulação}

O protocolo de superovulação utilizado ao longo dos quatro anos está representado na Figura 1. No primeiro dia, foi colocado um dispositivo intravaginal de silicone impregnado com 0,33 g de progesterona (CIDR ${ }^{\circledR}$, Zoetis, São Paulo, Brasil), trocado no dia sete por outro novo e mantido até o dia 17. Além disso, no dia sete foi realizada a aplicação de $0,24 \mathrm{mg}$ de cloprostenol de sódio (Sincrocio ${ }^{\circledR}$, Ourofino Saúde Animal, São Paulo, Brasil). A partir do dia 12 foram feitas aplicações de 256 mg de hormônio folículo estimulante (FSH) (Foltropin ${ }^{\circledR}$, Vetoquinol Saúde Animal Ltda., São Paulo, Brasil) em doses decrescentes por quatro dias em intervalos de 12 horas. No momento da retirada do dispositivo foi aplicado 200 UI de gonadotrofina coriônica equina (eCG) (Novormon $^{\circledR}$, Zoetis Ltda, São Paulo, Brasil) e 0,1 mg de hormônio liberador de gonadotrofina (GnRH) (Fertagyl ${ }^{\circledR}$, MSD Saúde Animal, São Paulo, Brasil) um dia antes da inseminação artificial.

Figura 1 - Esquema detalhado do protocolo de superovulação realizado em doadoras ovinas da raça Dorper ao longo de quatros anos (2017-2020).

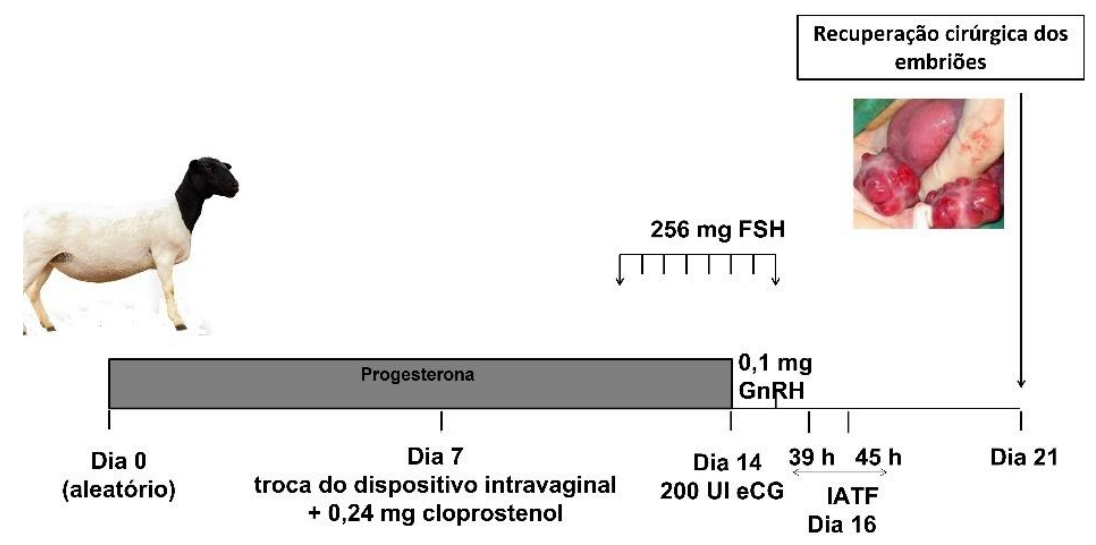

FSH: hormônio folículo estimulante; eCG: gonadotropina coriônica equina; GnRH: hormônio liberador de gonadotropina; IATF: Inseminação artificial em tempo fixo. Fonte: elaborado pelos autores (2021). 


\subsection{Coleta de sêmen, avaliação e Inseminação Artificial (IA)}

O sêmen foi adquirido de diferentes cabanhas de reprodutores de alto valor genético. O sêmen foi colhido um dia antes da inseminação artificial, avaliado e selecionado de acordo com a qualidade seminal (CBRA, 2013). Foi adicionado diluente comercial (Botu-bovi ${ }^{\circledR}$, Biotech Ltda, Botucatu-SP) e depois acondicionado em caixa térmica (Botu-flex ${ }^{\circledR}$, Biotech Ltda, BotucatuSP). A IA foi realizada por laparoscopia utilizando sêmen resfriado no volume de $0,3 \mathrm{~mL}$ em cada corno uterino na dose inseminante de $100 \times 10^{6}$ de espermatozoides, 36 e 42 horas após a retirada do dispositivo intravaginal. Foi utilizada uma pipeta de IA de pequenos ruminantes (WTA Ltda, Cravinhos, Brasil) para a deposição do sêmen diretamente na luz uterina de cada corno separadamente (Mckelvey, Robinson, Aitken \& Robertson, 1986).

\subsection{Coleta dos embriões}

O procedimento se iniciou com a tricotomia da região abdominal, seguida da aplicação intravenosa de xilazina $2 \%$ $(0,05 \mathrm{mg} / \mathrm{kg})$ e cetamina $(2 \mathrm{mg} / \mathrm{kg})$. A coleta dos embriões foi realizada por meio da técnica cirúrgica de laparotomia com exposição dos cornos uterinos cinco dias após a IA (Bruno-Galarraga et al., 2015). Cada corno uterino foi lavado com $30 \mathrm{~mL}$ de meio PBS (Nutricell, São Paulo, Brasil) utilizando uma sonda de Foley de silicone de duas vias número 10 (Rusch ${ }^{\circledR}$, EUA). O lavado foi acondicionado em placa de Petri $(100 \mathrm{~mm})$ e avaliado sob microscópio estereoscópico. Os embriões foram classificados com base na qualidade e grau de desenvolvimento (IETS, 2010). Após a seleção, os embriões foram mantidos em placa de Petri $(35 \mathrm{~mm})$ contendo meio holding (Nutricell, São Paulo, Brasil) à temperatura ambiente e protegidos contra a luminosidade até o momento da transferência para as receptoras. A ocorrência de aderência nas doadoras foi avaliada e classificada como: transitória (doadoras que foram acasaladas por monta natural posteriormente, tornaram-se gestantes, pariram uma cria viável e em alguns casos até retornaram para novos procedimentos de coleta) ou permanente (não ficaram gestantes após mais de três ciclos reprodutivos, ou seja, perda da habilidade reprodutiva).

\subsection{Receptoras de embriões}

As receptoras selecionadas $(\mathrm{n}=140)$ foram ovelhas de cruzamento da raça Santa Inês, com idade entre dois e cinco anos e escore corporal entre 2 e 3 (escala 1 - 5). Para cada doadora submetida ao protocolo, eram sincronizadas cinco receptoras. Estas foram submetidas à sincronização de estro um total de 283 vezes, sendo que 256 destas, receberam embriões. A utilização das receptoras era dependente do número de embriões disponíveis e do status ovariano no momento da transferência, que será descrito posteriormente.

O protocolo de sincronização iniciava cinco dias após o das doadoras, com a colocação do dispositivo intravaginal de silicone impregnado com $0,33 \mathrm{~g}$ de progesterona $\left(\mathrm{CIDR}^{\circledR}\right)$ de segundo uso, $500 \mathrm{UI}$ de eCG (Novormon ${ }^{\circledR}$ ) e 0,24 mg de cloprostenol de sódio $\left(\operatorname{Sincrocio}^{\circledR}\right)$ no dia 14 do protocolo. Em seguida, as ovelhas foram colocadas com o rufião previamente marcado na região do esterno com uma mistura de óleo de soja com tinta em pó. Deste modo, assim que apresentavam sinais de estro, as fêmeas eram marcadas pelo rufião e separadas das demais.

\subsection{Transferência dos embriões e diagnóstico de gestação}

Para o procedimento, as ovelhas receberam xilazina $2 \%(0,05 \mathrm{mg} / \mathrm{kg})$ seguido da tricotomia da região abdominal. Para identificar as receptoras aptas a receber o embrião, os ovários eram avaliados por laparoscopia com a condição da presença de pelo menos um corpo lúteo funcional com a coloração rósea escuro a vermelha em um dos ovários. A inovulação dos embriões foi realizada por técnica semi laparoscópica com a exposição de uma pequena porção do corno uterino ipsilateral ao corpo lúteo ativo. Todas as receptoras receberam apenas um embrião, de qualidade 1 ou 2, em estágio de mórula ou blastocisto. Após o procedimento, elas retornaram ao mesmo local para aguardar a confirmação da gestação. O diagnóstico de gestação ocorreu aos 40 
dias após o procedimento por via transretal utilizando transdutor linear de $5 \mathrm{MHz}$ (Chisson Eco $3^{\circledR}$, Kilumax, São Paulo, Brasil).

\subsection{Custos}

Foram levantados todos os custos diretos relacionados à MOTE. Não foram incluídos os custos indiretos, como os referentes a investimento e depreciação da estrutura e outros materiais da cabanha. Isto ocorreu pelo fato desta biotécnica poder ser realizada sem a necessidade de uma estrutura própria, já que os custos das instalações variam de uma propriedade para outra. Dessa maneira, os custos diretos foram os relativos a(o): protocolo hormonal, aquisição do sêmen, mão de obra do médicoveterinário, salário proporcional ao tempo utilizado pelo funcionário na preparação dos animais e com os procedimentos. Para o protocolo hormonal das doadoras e receptoras foram levantados os valores gastos em nota fiscal com os itens utilizados, calculando de maneira proporcional por cada indivíduo. Os valores acompanharam os praticados pelo mercado no momento da compra. Nos valores do protocolo hormonal, foram incluídas as ovelhas que realizaram o protocolo, mas que por falta de resposta reprodutiva ou falta de embrião para transferência não deram continuidade aos procedimentos.

O sêmen foi adquirido de diferentes cabanhas, com um valor que variou entre $\mathrm{R} \$ 400$ e $\mathrm{R} \$ 600$ reais, cobrado apenas das ovelhas que produziram ao menos um embrião viável. Quando foi utilizado sêmen de reprodutores da própria cabanha foi estimado o valor dos materiais utilizados para a coleta do sêmen e valor proporcional da mão de obra do médico-veterinário, variando de $\mathrm{R} \$ 31,00$ a $\mathrm{R} \$ 34,24$ por doadora ao longo dos anos.

Para o valor de mão de obra do médico-veterinário foi considerado o valor de $\mathrm{R} \$ 700,00$ praticado por profissionais da região, sendo calculado o valor por doadora coletada, excluindo as ovelhas que não responderam ao protocolo de superovulação ou apresentaram qualquer condição que não pudesse concluir a coleta e ficando por conta do médico-veterinário a aquisição do material de consumo. Já o valor de mão de obra do funcionário, foi calculado mediante a quantidade de horas proporcionais ao salário mensal, utilizadas com a preparação dos animais e dias dos procedimentos.

\subsection{Análise da capacitação profisssional}

Após o treinamento técnico no início do ano 1, o médico-veterinário foi adquirindo experiência ao longo dos anos a partir da execução e treinamento da MOTE. Para mensurar essa influência foram calculados os índices reprodutivos para cada um dos anos em que a técnica foi realizada no mesmo rebanho e pelo mesmo médico-veterinário. Esses índices foram taxas de estruturas totais por coleta e a quantidade de embriões transferidos por doadora, taxa de gestação das receptoras após a inovulação dos embriões e aderências uterinas nas doadoras resultantes dos procedimentos cirúrgicos.

\subsection{Margem bruta da MOTE}

A margem bruta é um parâmetro de rentabilidade em uma operação econômica e indica qual a porcentagem de lucro obtido em um investimento. Se ela for positiva, o produtor consegue ao menos saldar o investimento e no caso de ser negativa, há a necessidade de captação de outras fontes de recursos para a manutenção do processo produtivo (CNA Brasil, 2021). Dessa maneira, a margem bruta correspondeu ao lucro direto obtido exclusivamente através da utilização da MOTE no rebanho. Foram calculados os custos por embrião produzido, custos da gestação e custos por cordeiro nascido, incluindo os custos diretos com a técnica e o valor médio de venda dos produtos, mostrando a proporção do valor da MOTE no custo por gestação e depois na venda dos animais.

\subsection{Estatística}

Para a análise estatística foi usado o software Bio Estat 5.0 (Belém, Brasil), considerando o nível de significância de 5\% $(\mathrm{P}<0,05)$. Teste de Shapiro Wilk foi aplicado para verificar normalidade. Variáveis não paramétricas foram analisadas por teste de 
Kruskal-Wallis seguido do teste de Dunn, ou teste de qui-quadrado e teste exato de Fisher.

\section{Resultados}

No que se refere aos parâmetros reprodutivos (Tabela 1), o número médio de estruturais totais recuperadas bem como a média de embriões transferidos por coleta apresentaram diferença $(\mathrm{P}<0,05)$ entre os anos. Para ambas as variáveis, o valor máximo foi atingido no Ano 4, em patamares distintos do Ano 1. Já para gestações, taxa de aderência permanente e transitória não houve diferença $(\mathrm{P}>0,05)$, ao passo que a proporção de receptoras sincronizadas que receberam embrião apresentou diferença significativa após o primeiro ano.

Tabela 1. Parâmetros reprodutivos (média \pm SEM ou \%) obtidos com a adoção da MOTE ao longo de quatro anos em rebanho comercial ovino da raça Dorper

\begin{tabular}{lccccc}
\hline Variáveis & Ano 1 & Ano 2 & Ano 3 & Ano 4 & Total/Média \\
\hline Número de doadoras/coletas & 14 & 23 & 14 & 14 & 65 \\
Número de estruturas totais recuperadas & 34 & 137 & 64 & 115 & 350 \\
Média de estruturas/doadora & $2,4 \pm 0,7^{\mathrm{a}}$ & $6,0 \pm 1,0^{\mathrm{a}, \mathrm{b}}$ & $4,6 \pm 1,0^{\mathrm{a}, \mathrm{b}}$ & $8,2 \pm 2,1^{\mathrm{b}}$ & $5,3 \pm 2,4$ \\
Número de embriões transferidos & 24 & 103 & 59 & 85 & 271 \\
Média de embriões transferidos/doadora & $1,7 \pm 0,5^{\mathrm{a}}$ & $4,5 \pm 0,7^{\mathrm{b}}$ & $4,2 \pm 0,9^{\mathrm{a}, \mathrm{b}}$ & $6,1 \pm 1,8^{\mathrm{b}}$ & $4,1 \pm 1,8$ \\
Número de gestações (receptoras) & 6 & 41 & 28 & 34 & 109 \\
Taxa de gestação (\%) & 25,0 & 39,8 & 47,5 & 48,6 & 40,2 \\
Receptoras recebendo embrião (\%) & $34,3^{\mathrm{a}}$ & $89,6^{\mathrm{b}}$ & $84,3^{\mathrm{b}}$ & $94,0^{\mathrm{b}}$ & 75,5 \\
Número de cordeiros nascidos & 5 & 36 & 25 & 30 & 96 \\
Número de aderências permanentes & 2 & 0 & 0 & 0 & 2 \\
Taxa de aderência permanente (\%) & 14,3 & 0 & 0 & 0 & 3,6 \\
Número de aderências transitórias & 1 & 1 & 2 & 0 & 4 \\
Taxa de aderência transitória (\%) & 7,1 & 4,3 & 14,3 & 0 & 6,4 \\
\hline
\end{tabular}

${ }^{\mathrm{a}, \mathrm{b}}$ Diferença entre anos para médias e taxas $(\mathrm{P}<0.05)$. Fonte: Autores $(2021)$.

Os custos diretos da MOTE apresentados na Tabela 2 mostram as variações no custo do sêmen que foi substancialmente maior no primeiro ano, já que no início do experimento o material foi adquirido de fonte externa e nos anos subsequentes já havia machos com alto valor genético na propriedade aptos a serem coletados e inclusos na reprodução. No Ano 2 houve maior custo com mão de obra, visto que a quantidade de animais envolvidos no experimento foi sensivelmente maior, demandando mais tempo de serviço (Figura 2). 
Tabela 2. Custos diretos em reais $(\mathrm{R} \$)$ relacionados à adoção da MOTE ao longo de quatro anos em rebanho comercial ovino da raça Dorper

\begin{tabular}{ccccccc}
\hline Ano & $\begin{array}{c}\text { Protocolo } \\
\text { por doadora }^{*}\end{array}$ & $\begin{array}{c}\text { Protocolo por } \\
\text { receptora* }\end{array}$ & Sêmen & $\begin{array}{c}\text { Mão de obra } \\
\text { veterinário*** }\end{array}$ & $\begin{array}{c}\text { Mão de obra } \\
\text { funcionário }\end{array}$ & $\begin{array}{c}\text { Custo direto } \\
\text { total ano }\end{array}$ \\
\hline 1 & 289,55 & 27,13 & 305,00 & 700,00 & 563,55 & $20.586,05$ \\
2 & 314,01 & 26,94 & 107,07 & 700,00 & 926,71 & $29.809,64$ \\
3 & 237,37 & 27,48 & 135,00 & 700,00 & 563,55 & $17.500,14$ \\
4 & 502,09 & 29,12 & 206,30 & 700,00 & 563,55 & $22.319,14$ \\
\hline
\end{tabular}

*Valor relativo aos custos de todas as doadoras/receptoras que receberam o protocolo hormonal mesmo que não tenha respondido ao tratamento, dividido pelos animais que foram utilizados; ${ }^{* *}$ Valor proporcional por doadora que produziu ao menos um embrião; ${ }^{* * *}$ Valor relativo aos procedimentos realizados em cada ano, cobrado por doadora coletada; ${ }^{* * * *}$ Valor relativo ao tempo necessário para a realização de todos os procedimentos e preparação dos animais. Fonte: Autores (2021).

Figura 2 - Proporção de cada custo direto no valor total referente à aplicação da MOTE em rebanho ovino comercial no decorrer de quatro anos.

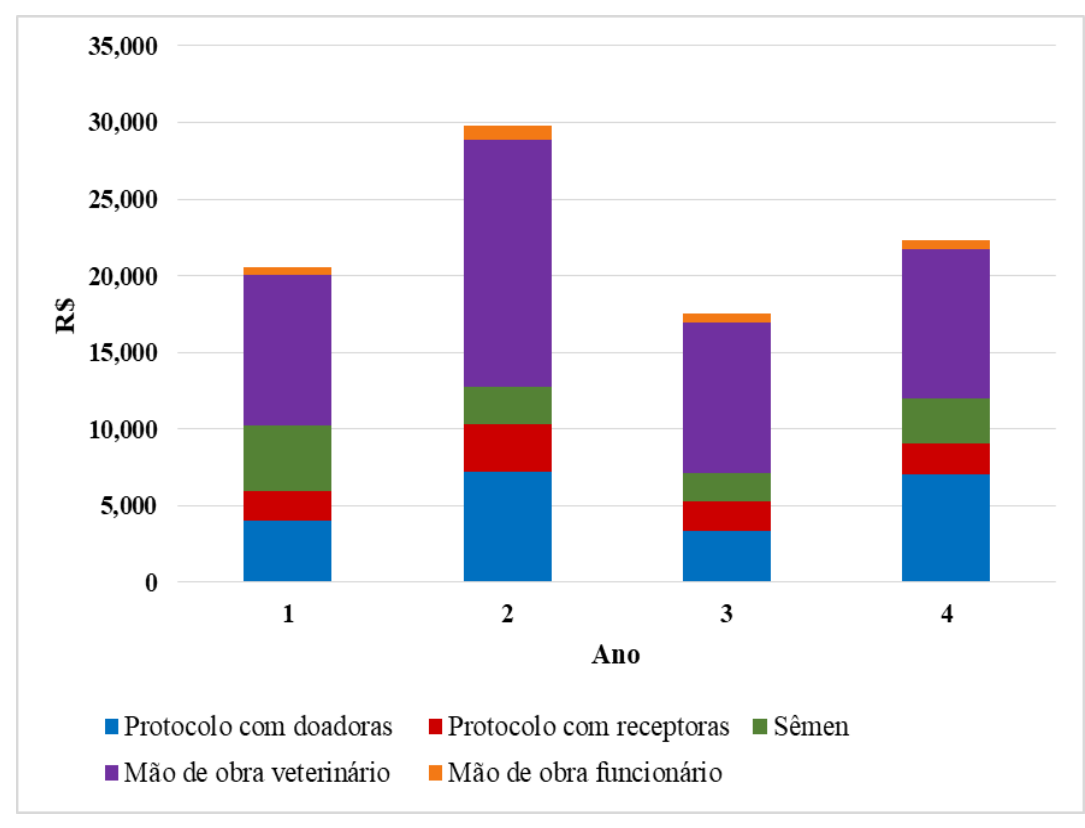

Fonte: Autores (2021).

Quanto aos custos totais diretos por gestação, foi observado um salto no Ano 2, a despeito da economia na obtenção do sêmen. Este salto foi impulsionado por maior efetividade na produção de estruturas totais que resultou em incremento na transferência de embriões dada a expertise adquirida, bem como a maior quantidade de animais envolvidos no ano. Tal incremento recuou nos anos subsequentes, no entanto, mantendo sempre os custos por gestação controlados diante da realidade advinda do primeiro ano (Tabela 3). 
Tabela 3. Margem bruta (R\$) por gestação da cabanha com a adoção da MOTE

\begin{tabular}{cccccc}
\hline Ano & $\begin{array}{c}\text { Custo por embrião } \\
\text { produzido }\end{array}$ & Custo por gestação & $\begin{array}{c}\text { Custo por cordeiro } \\
\text { nascido }\end{array}$ & Valor médio de venda & Margem Bruta \\
\hline 1 & 857,75 & $3.431,01$ & $4.117,21$ & $3.160,00$ & $-271,01$ \\
2 & 289,41 & 727,04 & 828,04 & $3.042,00$ & $2.314,56$ \\
3 & 296,61 & 625,00 & 700,00 & $3.971,00$ & $3.346,33$ \\
4 & 262,57 & 656,45 & 743,97 & $3.818,00$ & $3.161,11$ \\
\hline
\end{tabular}

Fonte: Autores (2021).

\section{Discussão}

O presente estudo avaliou os parâmetros reprodutivos da MOTE a partir dos índices de treinamento e capacitação profissional do médico-veterinário, custos relativos à implementação da biotecnologia e valores comerciais de venda dos animais. Bari et al. (2001), demonstraram que a quantidade de estruturas recuperadas ao longo de coletas sucessivas se manteve semelhante ao longo dos procedimentos nos mesmos animais e após um aperfeiçoamento da técnica foi possível observar discreta melhora. Porém, Bruno-Galarraga et al. (2014), observaram que a quantidade de estruturas totais sofreu um decréscimo ao longo dos procedimentos. Diferentemente do que ocorreu no estudo citado, a taxa de recuperação do Ano 1 foi muito inferior às demais e a partir daí os resultados foram apresentando evolução, demonstrando dessa maneira que se tratava de um período de treinamento e aperfeiçoamento do técnico. A ligeira queda observada no Ano 3 foi provavelmente decorrente das variações individuais nas respostas superovulatórias (Bartlewski et al., 2016).

Como consequência do aumento da quantidade de embriões coletados, houve uma diminuição significativa na quantidade de receptoras sincronizadas que ficaram sem receber embrião no decorrer dos anos. No primeiro ano da utilização da MOTE, apenas $34,3 \%$ das receptoras sincronizadas receberam embrião, seguido por $89,6 \%, 84,3 \%$ e $94,0 \%$ nos anos seguintes respectivamente. De acordo com Galli et al. (2003) a manutenção de receptoras na propriedade sem estarem produzindo é uma fonte importante de gasto financeiro com a biotecnologia.

A ocorrência de aderências do trato reprodutivo das ovelhas está relacionada com a reprodutibilidade da técnica devido a manipulação do útero (Cognie, 1999; Bruno-Galarraga et al., 2014). As ocorrências de aderências permanentes ou transitórias não apresentaram diferença significativa ao longo dos anos, porém, a ocorrência de perda da habilidade reprodutiva permanente foi observada apenas no primeiro ano em 14,3\% dos animais e não foi mais registrada nos anos seguintes. Este fato evidenciou a relação desse tipo de aderência com o treinamento do técnico.

As taxas de gestação das receptoras após a transferência dos embriões não apresentaram diferença significativa ao longo dos anos, variando de 25,0\% a 48,6\% (P>0,05) do Ano 1 ao 4. De acordo com Bari, Khalid, Haresign, Murray e Merrell (2003), tal feito está relacionado a fatores do próprio embrião como a qualidade e estágio de desenvolvimento no dia da coleta e fatores das receptoras como idade, partos anteriores e número de corpos lúteos no momento da transferência, não havendo nesse caso influência da experiência do médico-veterinário. Vale ressaltar que no presente estudo as receptoras apenas recebiam embriões caso apresentassem corpo lúteo funcional.

Em nossa pesquisa, de acordo com os valores que compreendem os custos diretos da MOTE ao produtor, os que apresentaram maior variabilidade foram o protocolo hormonal e o preço do sêmen. No Ano 3, devido a falta do FSH no mercado nacional, foi necessário adquirir medicamentos próximos a data de vencimento e, como consequência, conseguiu-se preços inferiores aos praticados normalmente no mercado. Ao contrário, no Ano 4, após o início da pandemia de SARS-Cov-2, foi observado um elevado acréscimo no valor de tal hormônio. Quanto ao sêmen, o valor gasto no primeiro ano foi o mais elevado em 
comparação aos demais pois com o melhoramento genético do rebanho a partir dos produtos da MOTE foi sendo possível utilizar os reprodutores da própria cabanha que tiveram a partir daí um aumento no ganho genético, diminuindo assim a quantidade de sêmen adquirido de outras criações.

Ao mesmo tempo, o fator que representou maior participação no valor total do custo da técnica para o produtor foi a mão de obra do médico-veterinário. Este fato evidencia a necessidade do treinamento e habilidade do profissional para o retorno financeiro da MOTE. Em relação ao custo por embrião produzido, gestação e cordeiro nascido, houve uma diminuição de aproximadamente cinco vezes ao final do estudo se comparado ao primeiro ano, apesar do aumento nos custos referentes aos protocolos hormonais. Essa redução pode ser reflexo da maior experiência adquirida pelo médico-veterinário ao longo dos anos, gerando melhores índices reprodutivos de forma geral. Isto pode ser observado no melhor aproveitamento das doadoras e receptoras, que são responsáveis por aproximadamente metade dos custos referentes a um programa de transferência de embriões, segundo Simplício, Freitas e Fonseca (2007). Dessa maneira, foi possível observar um aumento da eficiência da técnica ao longo dos anos.

Ribeiro, Galvão, Thatcher e Santos (2012) demonstraram que a estratégia adotada para a reprodução é o ponto chave para a dinâmica do rebanho, a partir do lucro ou prejuízo que esse manejo pode proporcionar. No presente estudo, a partir da MOTE, foi possível acelerar o melhoramento genético na cabanha, aumentando o valor médio de venda dos animais, se comparados o primeiro e último ano do estudo. É importante observar que somente no primeiro ano, devido ao início do treinamento técnico, houve margem bruta negativa, cenário que foi revertido a partir do segundo ano.

\section{Conclusão}

Em conclusão, o treinamento técnico do médico-veterinário ao longo dos quatro anos foi capaz de diminuir os custos e aumentar a eficiência da MOTE em rebanho ovino da raça Dorper, acelerando o melhoramento genético na cabanha. Este tipo de estudo pode ser determinante para a decisão de aplicação desta biotecnologia da reprodução pelo produtor rural. Outro ponto importante é que este estudo foi realizado em São Paulo, Brasil, e sua utilização como modelo de simulação em outras localidades pode sofrer influências que alterem os seus resultados e aplicabilidade. Sugere-se a realização de pesquisas futuras focando nos custos diretos e indiretos relacionados à utilização e viabilidade econômica das diferentes biotecnologias reprodutivas, tanto para a espécie ovina, como para as demais espécies de produção. Estudos com análises econômicas são escassos, mas essenciais para a maior difusão destas biotécnicas a nível comercial.

\section{Agradecimentos}

Este estudo foi financiado pelo Conselho Nacional de Desenvolvimento Científico e Tecnológico (CNPq) e Coordenação de Aperfeiçoamento de Pessoal de Nível Superior (CAPES) - Código de Financiamento 001, por meio de bolsas de estudo. Joanna Maria Gonçalves Souza-Fabjan é bolsista de produtividade do CNPq e Jovem Cientista do Nosso Estado pela FAPERJ.

\section{Referências}

Alves, L. G. C., Osório, J. C. S., Fernandes, A. R. M., Ricardo, H. A. \& Cunha, C. M. (2014). Produção de carne ovina com foco no consumidor. Enciclopédia Biosfera, 10(18), 2399-2014.

Baltrame, R. T., Quirino, C. R., Barioni, R. G. \& Lima, V. F. M. H. (2010). Simulação e análise econômica da produção in vivo e in vitro de embriões. Pesquisa Agropecuária Brasileira, 45(12).

Bari, F., Khalid, M., Wolf, B., Haresign, W., Murray, A. \& Merrell, B. (2001). The repeatability of superovulatory response and embryo recovery in sheep. Theriogenology, 56(1), 147-155.

Bari, F., Khalid, M., Haresign, W., Murray, A. \& Merrell, B. (2003). Factors affecting the survival of sheep embryos after transfer within a MOET program. Theriogenology, 59, 1265-1275. 
Bartlewski, P. M., Seaton, P., Oliveira, M. E. F., Kridli, R. T., Murawsky, M. \& Schwarz, T. (2016). Intrinsic determinants and predictors of superovulatory yields in sheep: Circulating concentrations of reproductive hormones, ovarian status, and antral follicular blood flow. Theriogenology, 86(1), 130-143.

Baruselli, P. S., Catussi, B. L. C., Abreu, L. A., Elliffi, F. M., Silva, L. G. \& Batista, E. O. S. (2019). Challenges to increase the AI and ET markets in Brazil. Animal Reproduction, 16(3), 364-375.

Brasil, O. O., Moreira, N. H., Ramos, A. F. (2014). Situação atual da superovulação em ovinos. Revista Brasileira de Reprodução Animal, $38(3)$, 147-153.

Bruno-Galarraga, M., Cueto, M., Gibbons, A. E., Pereyra-Bonnet, F., Catalano, R. \& Gonzalez-Bulnes, A. (2014). Repeatability of superovulatory response to successive FSH treatments in Merino sheep. Small Ruminant Research, 120(1), 84-89.

Bruno-Galarraga, M., Cueto, M., Gibbons, A., Pereyra-Bonnet, F., Subiabre, M. \& Gonzalez-Bulnes, A. (2015). Preselection of high and low ovulatory responders in sheep multiple ovulation and embryo transfer programs. Theriogenology, 84, 784-790.

Cognie, Y. (1999). State of the art in sheep-goat embryo transfer. Theriogenology, 51(1), 105-116.

CBRA (2013). COLÉGIO BRASILEIRO DE REPRODUÇÃO ANIMAL. Manual para exame andrológico e avaliação de sêmen animal (3a ed., pp. 49-104).

Fonseca, J. F., Oliveira, M. E. F., Brandão, F. Z., Batista, R. T. P., Garcia, A. R., Bartlewski, P. M. \& Souza-Fabjan, J. M. G. (2019). Non-surgical embryo transfer in goats and sheep: the Brazilian experience. Reproduction, Fertility and Development, 31(1), 17-26.

Galli, C., Duchi, R., Crotti, G., Turini, P., Ponderato, N., Colleoni, S., Lagutina, I. \& Lazzari, G. (2003). Bovine embryo technologies. Theriogenology, 59(2), 599. 616.

Gerger, R.P.C., Rossetto, R., Ribeiro, E.S., Ortigari, I., Zago, F. C., Aguiar, L.H., Costa, U.M., Lopes, R. F. F., Ambrósio, C. E., Miglino, M. A., Rodrigues, J. L., Forell, F., Bertolini, L. R. \& Bertolini, M. (2017). Impact of cumulative gain in expertise on the efficiency of handmade cloning in cattle. Theriogenology, 95, 2432.

Heikkila, A. M. \& Peippo, J. (2012). Optimal utilization of modern reproductive technologies to maximize the gross margin of milk production. Animal Reproduction Science, 132, 129-138.

Mckelvey, W. A. C., Robinson, R. P., Aitken, R. P. \& Robertson, I. S. (1986). Repeated recoveries of embryos from ewes by laparoscopy. Theriogenology, 25(6), $855-865$.

Phillips, P. E. \& Janke, M. M. (2016). Embryo transfer: Techniques, donor and recipients. Veterinary Clinics of North America: Food Animal Practice, 32(2), 365385.

Ribeiro, E. S., Galvão, K. N., Thatcher, W. W., \& Santos (2012). Economics aspects of applying reproductive technologies to dairy herds. Animal Reproduction, $9(3), 370-387$.

Simplício, A. A.; Freitas, VJ de F.; da Fonseca, J. F. (2007). Biotécnicas da reprodução como técnicas de manejo reprodutivo em ovinos. Revista Brasileira de Reprodução Animal, 31(2), 234-246.

IETS (2020). Newsletters.

Zhu, J., Moawad, A. R., Wang, C. Y., Li, H. F., Ren, J. Y. \& Dai, Y. F. (2018). Advances in in vitro production of sheep embryos. International Journal of Veterinary Science and Medicine, 6, 15-26.

https://www.cnabrasil.org.br/custo-de-produc\%CC\%A7a\%CC\%83o-e-ana\%CC\%81lise-econo\%CC\%82mico-financeira. Acessado em 5 de junho de 2021. 